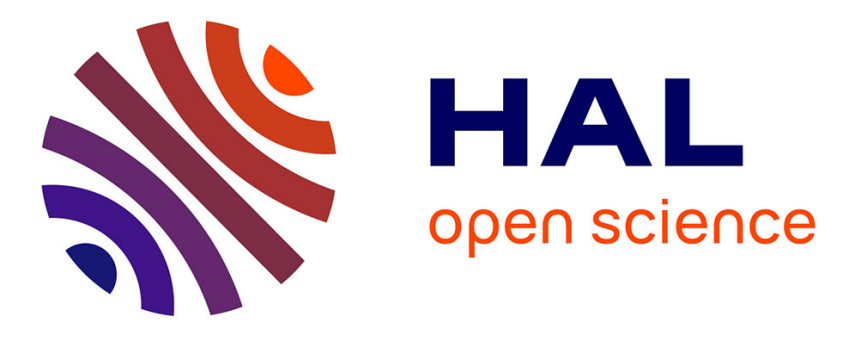

\title{
On the Suitability of Elekta's Agility 160 MLC for Tracked Radiation Delivery: Closed-loop Machine Performance
}

M Glitzner, S P M Crijns, Baudoin Denis de Senneville, J W Lagendijk, B W Raaymakers

\section{To cite this version:}

M Glitzner, S P M Crijns, Baudoin Denis de Senneville, J W Lagendijk, B W Raaymakers. On the Suitability of Elekta's Agility 160 MLC for Tracked Radiation Delivery: Closed-loop Machine Performance. Physics in Medicine and Biology, 2015, 60 (5), pp.2005-2017. 10.1088/0031-9155/60/5/2005/meta . hal-01578210

\author{
HAL Id: hal-01578210 \\ https://hal.science/hal-01578210
}

Submitted on 28 Aug 2017

HAL is a multi-disciplinary open access archive for the deposit and dissemination of scientific research documents, whether they are published or not. The documents may come from teaching and research institutions in France or abroad, or from public or private research centers.
L'archive ouverte pluridisciplinaire $\mathbf{H A L}$, est destinée au dépôt et à la diffusion de documents scientifiques de niveau recherche, publiés ou non, émanant des établissements d'enseignement et de recherche français ou étrangers, des laboratoires publics ou privés. 


\title{
On the Suitability of Elekta's Agility 160 MLC for Tracked Radiation Delivery: Closed-loop Machine Performance
}

\author{
M Glitzner ${ }^{1}$, S P M Crijns ${ }^{1}$, B Denis de Senneville ${ }^{1,2}$, \\ J J W Lagendijk ${ }^{1}$, B W Raaymakers ${ }^{1}$ \\ ${ }^{1}$ Department of Radiotherapy, University Medical Center Utrecht, Heidelberglaan \\ 100, 3584 CX Utrecht, the Netherlands \\ ${ }^{2}$ Mathematical Institute of Bordeaux, University of Bordeaux, 33405 Talence Cedex, \\ France \\ E-mail: m.glitzner@umcutrecht.nl
}

\begin{abstract}
.
For motion adaptive radiotherapy, dynamic multileaf collimator (dMLC) tracking can be employed to reduce treatment margins by steering the beam according to the organ motion.

Until now, the Elekta Agility $160 \mathrm{MLC}$ has hitherto not been evaluated for its tracking suitability under physiologic conditions. Both dosimetric performance and latency are key figures and need to be assessed generically, independent of the used motion sensor.

In this paper, we propose to use harmonic functions directly fed to the MLC to determine its latency during continuous motion. Furthermore, a control variable is extracted from a camera system and fed to the MLC. Using this setup, film dosimetry and subsequent $\gamma$ statistics are performed, evaluating the response when tracking magnetic resonance imaging (MRI)-based physiologic motion in a closed-loop.

The delay attributed to the MLC itself was shown to be a minor contributor to the overall feedback chain as compared to the impact of imaging components such as MRI sequences. Delay showed a linear phase behaviour of the MLC employed in continuously dynamic applications, which enables a general MLC-characterization. Using the exemplary feedback chain, dosimetry showed a vast increase in pass rate employing $\gamma$ statistics.

In this early stage, the tracking performance of the Agility using the test bench yielded promising results, making the technique eligible for the translation to tracking using clinical imaging modalities.
\end{abstract}

Keywords: MLC, tracking, system response, delay analysis, band-limited, linear-phase, physiologic motion

Submitted to: Phys. Med. Biol. 


\section{Introduction}

In an attempt to further limit radiation exposure of healthy tissue, real-time tracking has been evolved recently. Potentially, using tracking, the planning target volume (PTV) can be decreased significantly. Treatment margins added to account for set-up and motioninduced misalignments can be compensated using image guided radiotherapy (IGRT), while dose-painting the actual tumor volumes retaining high conformity with intensity modulated techniques(Giraud et al. 2006). A review of image guided radiotherapy can be found in (Ruan et al. 2011).

For multileaf collimator (MLC) tracking, several concepts have been demonstrated for main manufacturers of linac hardware (Tacke et al. 2010, Falk et al. 2012). Multiple position extraction techniques are utilized to assess tumor motion indirectly via surrogates, or directly via fiducials.

In order to obtain geometric organ position, mostly surrogate based positioning has been in use, amongst them optical positioning systems (Sawant et al. 2009) and electromagnetic transponders (Smith et al. 2009) (Real-time Position Management (RPM) system and Calypso, Varian Medical Systems Inc., Palo Alto, CA, USA).

Recently, the first clinical application utilized the latter modality on a prostate case (Keall et al. 2014). Moreover, tracking signals have been extracted from $\mathrm{kV}$ and MV imaging data (Berbeco et al. 2005, Cerviño et al. 2009) and recent studies also investigated dMLC tracking with the use of real-time MRI-signal (Crijns, Raaymakers \& Lagendijk 2012, Yun et al. 2013).

To this day, the performance of real-time MLC tracking with Agility 160 MLCs (Elekta AB, Stockholm, Sweden) has not yet been explored. However, Davies et al. (2013) reported on virtually tracked volumetric intensity modulated arc therapy (VMAT) using initial synchronization between the delivery and a motion platform.

The aspiration of this paper is to assess the system performance of the MLC in a generic sense. For the application in an integrated tracking modality, knowledge of the time delay of an MLC is fundamental, as it is the most sensitive parameter determining the geometric error in motion compensation (Poulsen et al. 2010, Ruan 2010), parameterization of prediction filters and retrospective dose accumulation. The relative contribution of the MLC is needed for evaluating its feasibility in a feedback chain with clinical components such as in an MR-Linac system developed in our institution (Raaymakers et al. 2009, Lagendijk et al. 2014). Due to the bandlimited character of physiologic motion, the time shift between aperture application and mechanic execution is determined in a physiologically reasonable continuous motion regime. To legitimate a general definition of the delay, the MLC is tested for linear phase behaviour in this range of harmonics.

Additionally, an optical feedback chain consisting of fast imaging, image processing and $1 D$ aperture adaptation is prototyped which serves as a test bench for the extraction of timing characteristics of the MLC and for actual dosimetry. It can easily be adapted to other control variables, potentially sourced from other imaging techniques such 
as from magnetic resonance imaging (MRI), electromagnetic transponders or optical positioning surrogates. The final endpoint being radiation depositions in clinical applications, the dosimetric impact of the actual feedback loop is measured in response to a physiologic motion scenario. Tracked and untracked dose maps are compared with integral quantifiers, namely tight-radius $\gamma$-analysis and relative dose histograms, in order to rate the tracking performance without regarding for uncertainties such as from planning or from positioning.

\section{Methods}

The methods are divided into a general part comprising hardware and evaluation (sections 2.1 to 2.4) together with the description and measurements of the elements involved in open- and closed-loop MLC control (sections 2.5 to 2.7).

\subsection{MLC characteristics}

For all experiments, Elekta's Agility 160 MLC as part of a clinical Axesse (Elekta AB, Stockholm, Sweden) linear accelerator is used. The Agility features 80 leaf pairs and dynamic diaphragms to limit the aperture perpendicular to leaf travel direction. For reasons of data presentation and evaluation, an aperture of $20 \times 40 \mathrm{~mm}^{2}$ was applied, by separating all leaves by $40 \mathrm{~mm}$. The arising gap is then limited to $20 \mathrm{~mm}$ using the diaphragm. The leaf motion is in caudo-cranial (CC) direction, parallel the longer side of the rectangular aperture. The head is thus rotated to $90^{\circ}$. The aperture width of $40 \mathrm{~mm}$ is chosen to yield a global dose plateau for each measurement, i.e. a point of permanent irradiation. This is needed to obtain a reference measurement for the relative dosimetry, detailled in section 2.3.

\subsection{Tracking components}

The feedback loop comprised an optical path for object detection and a control loop which shifts the aperture in response to object motion. The optical path located the position of a binary image (target) mounted on a linearly (1D, along leaf motion direction) moving object. The target image contained a contrast-rich structure with sharp gradients (see figure $1 \mathrm{~b}$ ) in all directions in order to make it locatable by the image processing explained below. The camera was positioned in such a way, that the target image was moving in a fixed imaging plane perpendicular to the camera's optical axis. Secondly, the control loop polled the extracted object's position continuously and applied it in form of a rigid aperture translation (again $1 D$ in leaf motion direction) to the MLC system.

The in-house developed tracking software served two purposes, depicted in figure 1: image acquisition and processing and commanding the MLC. In order to obtain the position of the tracked object, a camera (C930e, Logitech, Apples, Switzerland) presents a videostream $(640 p x \times 480 p x \times 30 H z)$ containing the marked object and passes the 


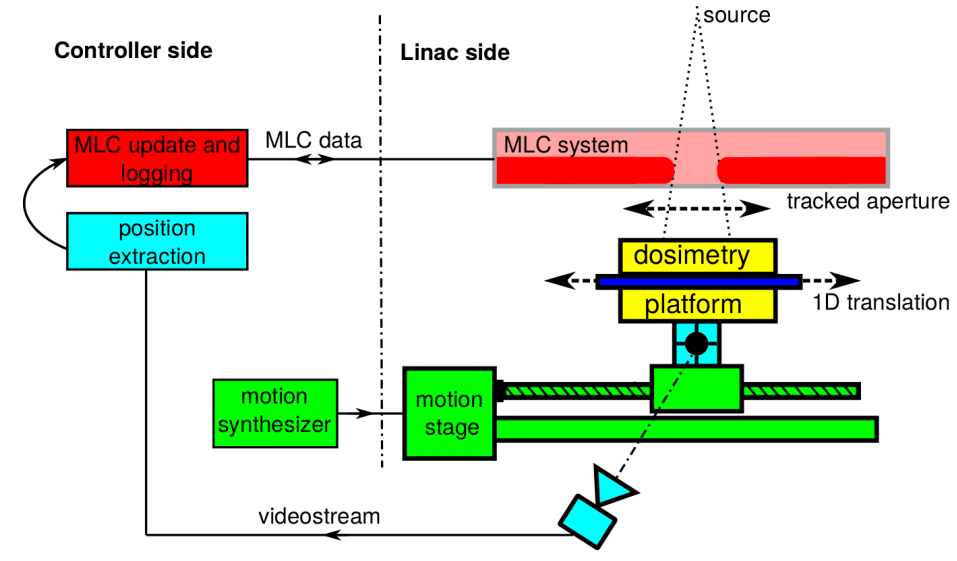

(a)

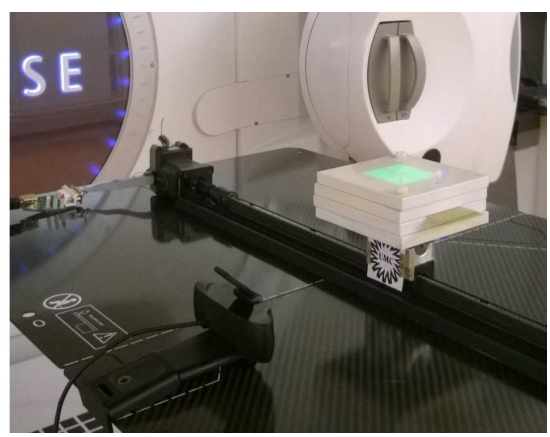

(b) Motion detection set-up

Figure 1. Schematic (a) and picture (b) of system components of the feedback loop, information flow and motions: The controller is commanding the MLC system and retrieving leaf positions from it. The position extraction locates the object position by processing a video stream from a camera. $1 D$ motion is synthesized and sent to the motion stage which translates the dosimetry stage and the attached target. See text for details.

stream to the image acquisition module. The camera was accessed by the computer vision library OpenCV (Bradski 2000). In order to obtain a reliable position extraction of the marked object by the subsequently described tracking algorithms, auto-focus features are disabled on camera-side.

In feedback experiments, the captured images are passed to a processing module without further conditioning.

The processing module extracts the pixel location of the marker based on a fast in-house minimum output sum of squared error (MOSSE) filter implementation (Bolme et al. 2010, Crijns, Kotte, Lagendijk \& Raaymakers 2012), tuned for working in a $64 \times 64 p x$ local region. Initially, before each experiment, geometric reference is established by a homing procedure in combination with the motion stage described below and a mean-free cross-correlation filtering. The latter extracts the position of an object marked with a known pattern from the captured images. Because of the relative slowness of this tracking method compared to the MOSSE filter, the correlation is only applied during homing. This algorithm transistion is necessary due to the local nature of the MOSSE filter; the detection region needs to be determined by the global cross-correlation algorithm first. In order to compare the processing performance, the runtimes of both algorithms were measured. Both filters operated on pixel pitch of $0.3 \mathrm{~mm} / \mathrm{px}$. The change in object position extracted by the image processing module were used as the control variable for the $1 D$ closed-loop aperture translation and the leaf positions of the translated aperture were applied to the Agility MLC control system via Ethernet with a cycle time of $40 \mathrm{~ms}$. Concurrently, the leaf positions provided by the MLC control system were continuously recorded.

All software was run via a Debian operating system on a computer equipped with 
$8 G B$ of memory and a 3.4GHz quad-core CPU (Core i5-2400, Intel, Santa Clara, CA, USA).

\subsection{Dosimetry}

The dosimetric platform is sketched in figure 1 showing a film sandwiched between two layers of water-equivalent solid build-up material of $2 \mathrm{~cm}$ thickness and a square surface of $10 \times 10 \mathrm{~cm}^{2}$ each. Relative dosimetry is performed using the radiosensitive GafChromic EBT3 film (Ashland Inc., Wayne, NJ, USA), with dimensions of about $10 \times 4 \mathrm{~cm}^{2}$. The longer side of the film is parallel to leaf travel direction and thus to the direction of motion.

Generally, the film set-up moved in a plane of $1 m S A D$ at isocenter. The object holding the film is referenced relative to the isocenter. In other words, an object in initial position ( $x=0$ in figure $2 \mathrm{a})$ is under an isocentric aperture. With each irradiation, a dose of $D=1000 \mathrm{MU}$ is delivered using a constant dose rate of $\dot{D}=400 \mathrm{MU} / \mathrm{min}$ at $6 M V$.

For planar $(2 D)$ dosimetry, the irradiated film is scanned using an Epson 10000XL flatbed scanner (Seiko Epson Corporation, Suwa, Japan). An automatic alignment method centers and rectifies the dose images using rigid transformations.

For $2 D$ evaluation, $\gamma$-statistics (Low et al. 1998) and dose difference test were used. The $2 D$ dose distribution are transformed to relative dose maps ranging between noise floor and dose plateau. To establish a stringent quantifier for the synthetic experiments, a tight $\gamma$-pass radius of $(1 \% / 1 \mathrm{~mm})$ was set. The dose-window to be evaluated is between $10 \%$ and $100 \%$ to reject low-dose regions, which we consider unimportant for rating the tracking performance.

\subsection{Phantom motion}

Motion is induced using a home-built $1 D$ translating stage. The linear positioning system (LPS) consists of an MDrive 17 (Schneider Electric Motion, Marlborough, CT, USA) driving a stage via a threaded rod (figure 1, green elements). The controller and drivers of the MDrive 17 are integrated and controllable via RS422. The motion stage carried the dosimetry set-up (cf. section 2.3) along with the optical target, detectable by the tracking algorithm (see figure 1b). In-house developed software was used to feed the stage with positioning data. The positioning data in the experiments were of two kind: synthetically generated sinusoidal curves and motion extracted from a temporally resolved liver position over multiple breathing cycles (see figure 2).

Sinusoids are applied to the motion stage for determining the overall closed loop response delay. Staying within a pseudo-continuous domain for the tracking system (the control variable does not strongly feature discrete step-behaviour), a parameterization of $(A, T)=(15 \mathrm{~mm}, 6 \mathrm{~s})$ was chosen, where $A$ is the amplitude and $T$ the duration of a sinusoid. 


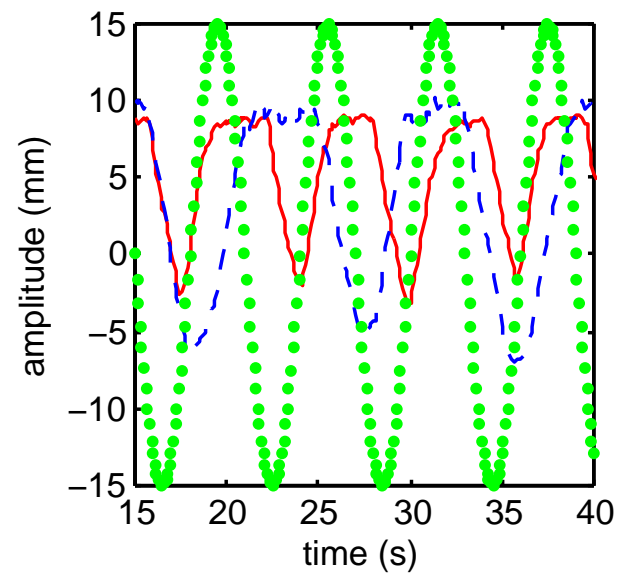

(a) time curves

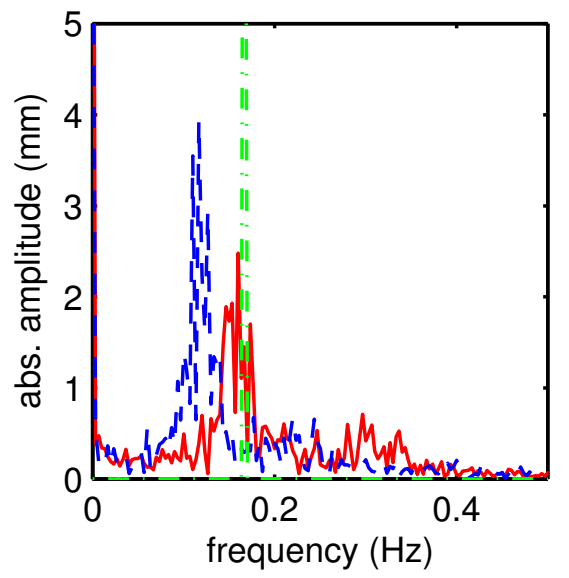

(b) single-sided spectra

Figure 2. Breathing motion data of two volunteers and the $(15 \mathrm{~mm}, 6 \mathrm{~s})$ sinusoid in temporal (a) and frequency (b) domain. Notably, most signal energy is under $0.3 \mathrm{~Hz}$. The spectrum is scaled to fit the physiologic signals.

Physiologic motion patterns were extracted from CC components of deformation vector fields (DVFs) in the liver, extracted from coronally acquired MRI with $10 \mathrm{~Hz}$ imaging frequency. The dosimetric response was evaluated based on these motion patterns in order to yield physiologically characteristic results. In order to present a continuous coordinate update to the motion controller, the data is upsampled by a factor of 2 to $20 \mathrm{~Hz}$ using MatlaB's (The Mathworks, Natick, MA, USA) standard reconstruction kernel (linear-phase Kaiser window filter). Consecutively, due to the relatively high noise level in the DVF, a $2.5 \mathrm{~Hz}$-Butterworth low-pass $(n=10)$ is applied to condition the raw motion signal before application to the motion stage. The noise level is caused by the echo planar imaging (EPI) nature of the acquisition and the consecutive DVF extraction by an algorithm using an optical flow signal model(Horn \& Schunck 1981). The cutoff frequency of the filter is chosen to leave physiologic components of the spectrum untouched. Thus, it solely filters higher-frequency noise induced by the imaging and motion estimation. The strong drop-off of the motion spectra over approximately $0.3 \mathrm{~Hz}$ in figure $2 \mathrm{~b}$ is an indicator for the frequency cut-off of the sampling field in the open-loop benchmark (cf. section 2.7).

\subsection{Lag determination}

The retardation of the entire system is broken down to its elements illustrated in figure 3. Two major experimental pathways have been followed throughout the experiment. Experiments regarding the MLC as an isolated element used the open-loop configuration, where the aperture is directly applied to the MLC (see figure 3 lower solid path). In contrast, performing closed-loop experiments (figure 3 upper solid path), the MLC was steered by the feedback control variable extracted by the tracking software.

From a control input on the left side of the schematic in figure 3, the signal 


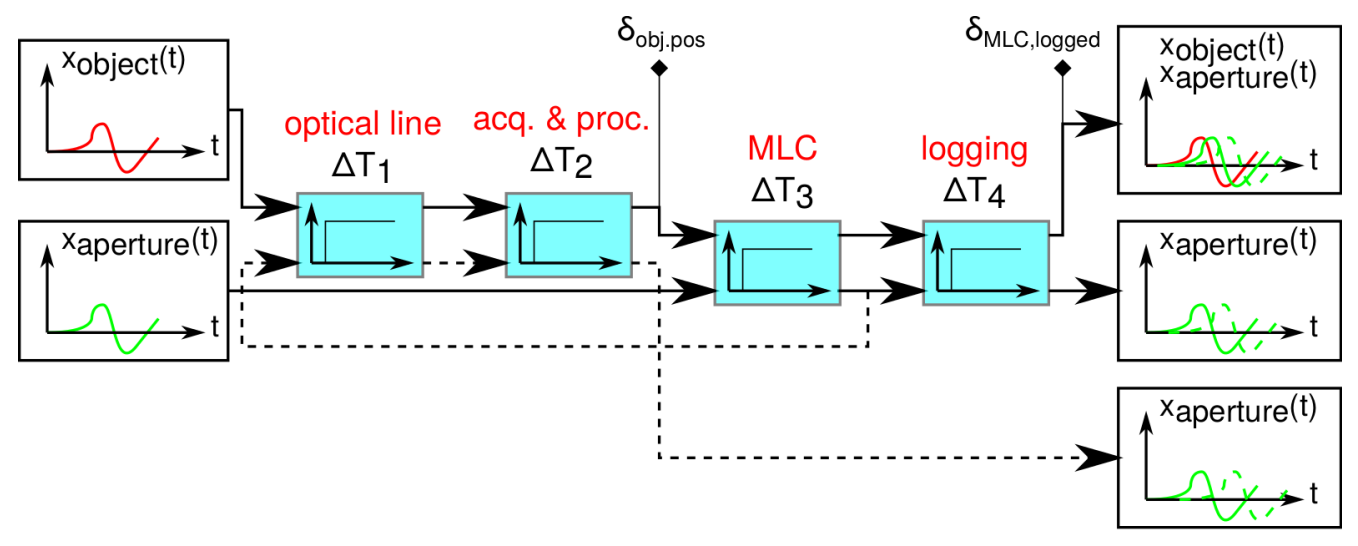

Figure 3. System lag components and experimental pathways in the feedback and open-loop configuration. The upper solid path emanating from the detected object motion represents closed-loop (or tracking) experiments. The other, lower solid paths show open-loop experiments with directly controlled MLC. For determination of the logging delay, the dashed pathway was employed. Diamonds indicate position data sampled. $\delta_{M L C, \text { logged }}$ and $\delta_{o b j . p o s}$ denote logging timing for MLC and object, respectively.

is sequentially passed through the respective processing steps which take up their individual times $\Delta T_{1,2,3,4}$. These elementary delays are regarded in detail subsequently in sections 2.6 and 2.7 .

\subsection{Closed tracking loop}

For the delay determination of the overall tracking system, the mean time elapsed between detection of the target and the aperture reaching that target has to be evaluated. This chain consists of several abstract elements which are specified in the following.

Regarding again figure 3, object motion has to be captured by the camera hardware. After the readout, data is buffered and streamed via USB and the camera driver module until it is passed to the OpenCV application programming interface (API). The arising time delay is called $\Delta T_{1}$. The received image is then processed subsequently in order to extract position information with the filtering algorithms presented in section 2.2. The runtimes of these filters, from the instance of grabbing the image until presenting the extracted position information to the MLC control system, are denoted $\Delta T_{2}$. After exposing the current target position to the software interface, the MLC system needs time to approach the desired position. This lag is caused by the runtime of the MLC control system and the electromechanical properties of the MLC and is called $\Delta T_{3}$.

Finally, for logging purposes, data is being received from the MLC side. Like the object position, the MLC position logging is delayed, taking $\Delta T_{4}$ to arrive at the control computer. 
For measuring the delay of the closed tracking loop, harmonics of the form

$$
x(t)=A \sin \left(\frac{2 \pi}{T}(t+\delta)\right)
$$

are applied to the motion stage (cf. section 2.4) and tracked by the feedback loop. Logging both position updates and actual leaf positions yields

$$
\Delta T_{\text {loop }}=\delta_{\text {obj.pos }}-\delta_{M L C, \text { logged }} .
$$

Since the position updates arrive and leaf positions arrive delayed by $\Delta T_{1}+\Delta T_{2}$ and $\Delta T_{4}$, respectively, the loop latency is to be corrected with

$$
\Delta T_{\text {loop,corr }}^{\prime}=\Delta T_{\text {loop }}+\left(\Delta T_{1}+\Delta T_{2}-\Delta T_{4}\right) .
$$

\subsection{Identification of lag elements}

Subsequently, the measurement of the system elements contributing to the overall delay of the feedback system are discussed.

Optical line delay $\Delta T_{1}$ is the integrated lag value between a physical change of lighting and its detection in software. Offering transistor-transistor logic (TTL) outputs, a green light emitting diode (LED) is connected to the parallel DB-25 interface. In two separate threads, the light is switched on irregularly and measured by means of (binary) light detection using the same image acquisition software as in the tracking application. Timestamping both incidents yields a mean delay between incident and detection (Schellen et al. 2013). The parallel port features a sufficiently fast reponse time in the $\mu s$-range (Koolwal 2009), sufficing the immediate switching requirement of this global time referencing step. The LED's electric dynamics is considered to be delay-free.

Acquisition and processing delay $\Delta T_{2}$ is the time it takes to grab an image from the OpenCV API and process and forward it to the MLC control. As the value is local runtime, the time $\Delta T_{2}$ is directly logged in each pass.

MLC delay or open-loop lag is the latency of the MLC system only. It is determined by commanding the aperture by a single-threaded program running at a fixed cycle time of $40 \mathrm{~ms}$ which corresponds to the real-time frame of the Agility MLC control system. Commands are sent to the MLC in each cycle. At the time of sending, the commanded position is recorded, as well as the actual leaf position retrieved by the logging stream introduced in section 2.2 .

To quantify the phase of the leaf positioning, an amplitude-period $(A, T)$ parameter field of harmonics is sampled by applying synthesized harmonics directly to the MLC. The sines are in the form of 1 , with parameter $A$ being in the domain $\{1,1.5,2,2.5$, $3\} \mathrm{cm}$ and $\mathrm{T}$ in $\{3,4,5, \ldots, 8\} \mathrm{s}$. Yielding pairs of sampled sine curves, the commanded 
and the observed, both curves are consequently fitted to a model as in 1 using MATLAB's Curve Fitting Toolbox. To obtain the dynamic MLC lag, the temporal displacement $\delta$ for both fits are related; as the observed state is delayed for the logging/subscription lag $\Delta T_{4}$, the measured phase difference is corrected for the subscription delay time $T_{4}$

$$
\Delta T_{3}=\delta_{M L C, \text { commanded }}-\delta_{M L C, \text { logged }}-\Delta T_{4} .
$$

Logging delay $\Delta T_{4}$ is indirectly measured by capturing the aperture light field (ALF) in an open-loop experiment. The ALF position is extracted using the tracking method descibed in 2.2. $\Delta T_{4}$ is then determined by fitting the ALF-mediated aperture position against the subscribed positions. The influence of $\Delta T_{1}$ and $\Delta T_{2}$ is regarded for by taking the previously determined values in the LED-delay experiment and in the image processing log data into account.

\section{Results}

The experiments on the feedback system are evaluated, starting from an elementary consideration of each element in the loop and its individual contribution to the feedback lag. Subsequently, the integral feedback lag is measured and its impact on the tracking experiment based on physiologic motion is shown.

\subsection{Elementary timings of the feedback system}

\begin{tabular}{|c|c|c|c|}
\hline Qty. & $\mu$ & $\widehat{\sigma}$ & remark \\
\hline- & $m s$ & $m s$ & - \\
\hline$\Delta T_{1}$ & 78.7 & 19.6 & multimodal \\
\hline$\Delta T_{2}$ & 20.6 & 1.7 & correlation $(100 \times 38 p x)$ \\
& 5 & 0.2 & MOSSE $(32 x 32 p x)$ \\
& 11.9 & 0.4 & MOSSE $(64 x 64 p x)$ \\
\hline$\Delta T_{3}$ & 15.9 & 6.6 & sampled parameter field (figure 4$)$ \\
\hline$\Delta T_{4}$ & 52 & - & one experiment (param.-fit) \\
\hline
\end{tabular}

Table 1. Results for average values of dead times $\Delta T_{1,2,3,4}$ with their estimated standard deviations $\widehat{\sigma}$.

Table 1 shows the resulting lag contributions for each component individually. Very prominent is the relatively long line feed time of the imaging system $\Delta T_{1}$. Additionally, its distribution deviates significantly. The various tracking algorithms unsurprisingly yield different lags in the $\Delta T_{2}$-estimation. The correlation, being a global filter, is the slowest. The subscription delay $\Delta T_{4}$ is extracted from a single parameter fit, thus no parameter statistics are available.

The open-loop delays $\Delta T_{3}$ field are depicted in figure 4a in a map which represents the parameter field. Although, a Gaussian distribution cannot be presumed, overlapping 


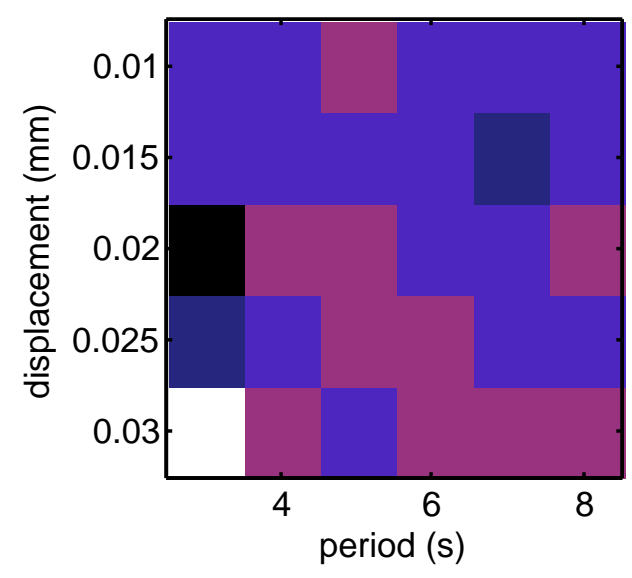

(a)

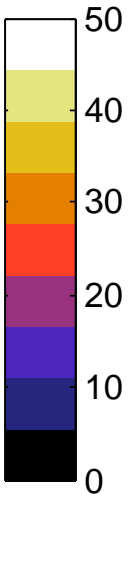

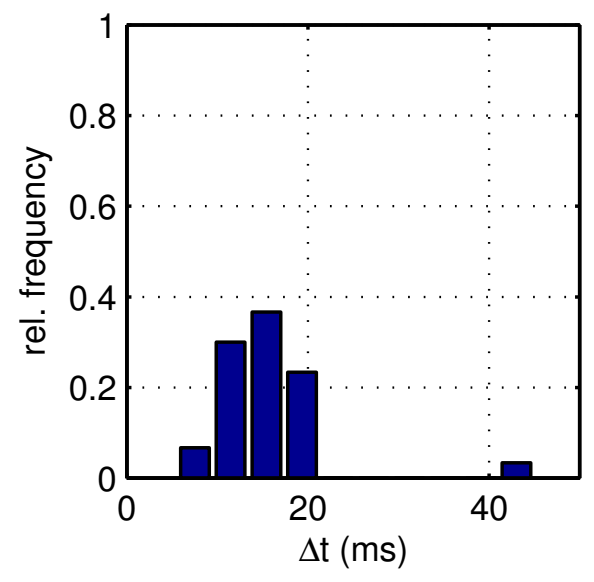

(b)

Figure 4. Open-loop MLC latencies for the sampled parameter field in (a). The distribution of the delay times is shown in (b).

median and mean $\Delta \bar{T}_{4}=\Delta \widetilde{T}_{4}=15.9 \mathrm{~ms}$ imply symmetry of the distribution with a estimated standard deviation of $\sigma=6.6 \mathrm{~ms}$. The minimal coefficient of determination of the obtained fits of the entire parameter field was $R_{\min }^{2}=99.63 \%$. The coefficients of determination indicate proper data fits and consequently significant lag extraction.

\subsection{Closed-loop feedback lag determination}

Figure 5 shows a section of the fitted data from object and aperture position. Both fits had $R^{2}>99.94 \%$. The extracted lag was determined to be $\Delta T_{\text {loop }, \text { corr }}^{\prime}=109.3 \mathrm{~ms}$, based on the relative phase between the fitted curves and after correction according to equation 3 .

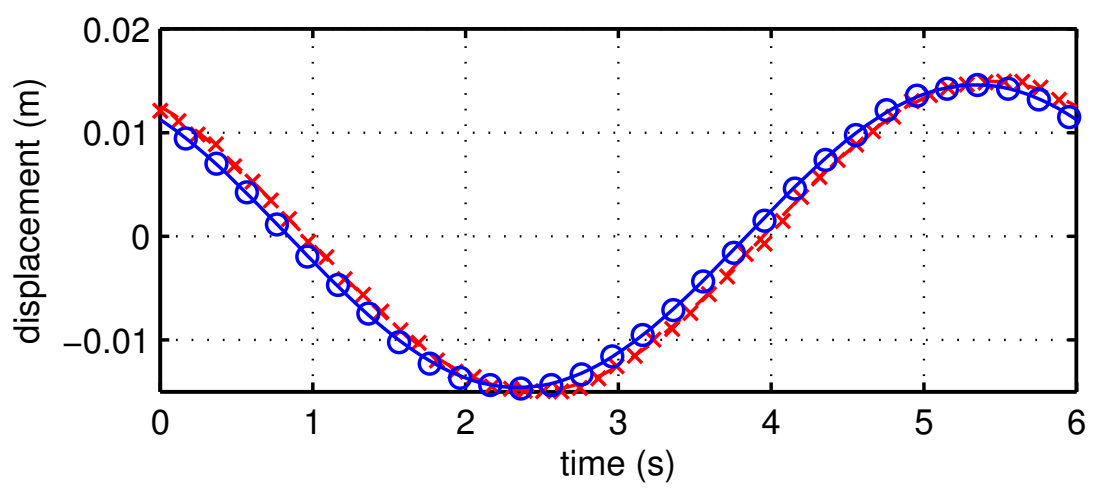

(a)

Figure 5. Closed-loop logging data and relative lag determination using fitted objection (solid-blue with $\circ$ data points) and aperture motion (red with $\times$-illustrated points. Solely every third data point is shown for clarity. 


\subsection{Film dosimetry}

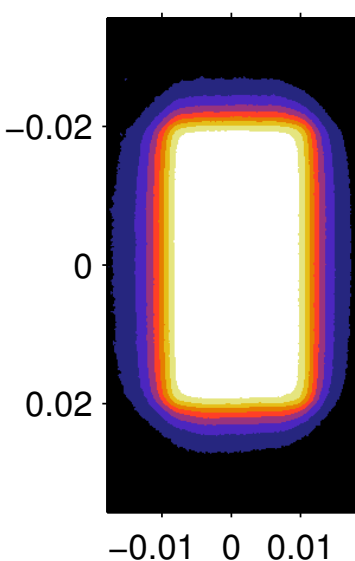

(a)

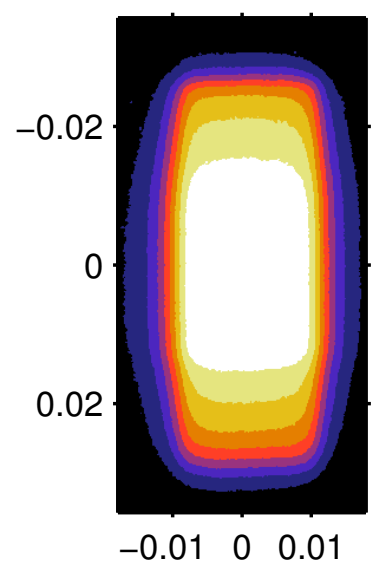

(b)

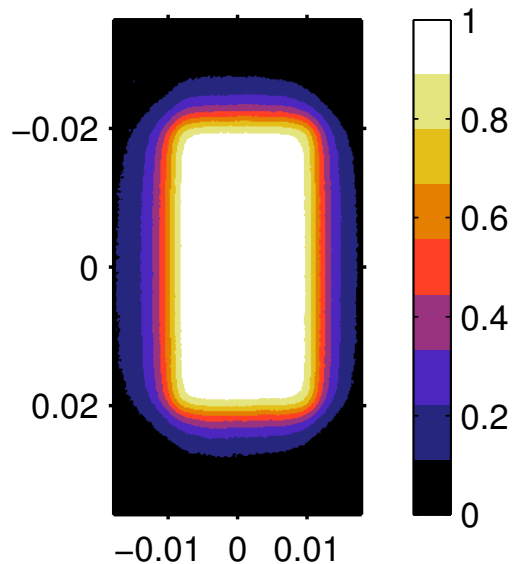

(c)

Figure 6. Normalized dose distributions under physiologic conditions: in a static (a), untracked dynamic (b) and tracked dynamic case (c). Leaf travel direction is the vertical direction. Axis units in $m$.

Irradiation under physiologic motion (blue traces in figure 2) yielded the normalized dose distribution in figure 6 . As expected, the untracked distribution in Figure $6 \mathrm{~b}$ shows significant smearing around the edges perpendicular to the leaf travel direction. Also lateral deviations are visible. Choosing an aperture relatively larger than the displacement of the tracked object $\left(w_{\text {aperture }} / 2>\max \{|\Delta x|\}\right)$ yields the anticipated visible dose plateau phase in the center of the distribution.

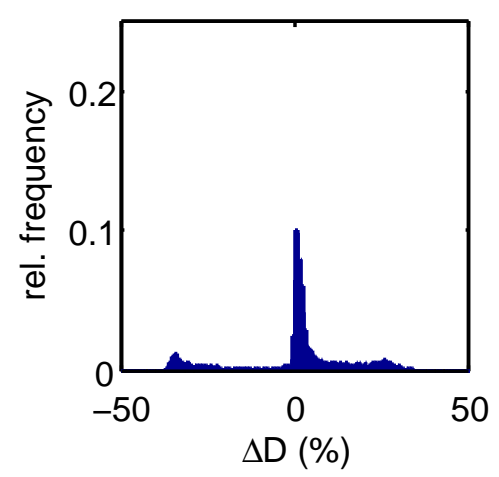

(a)

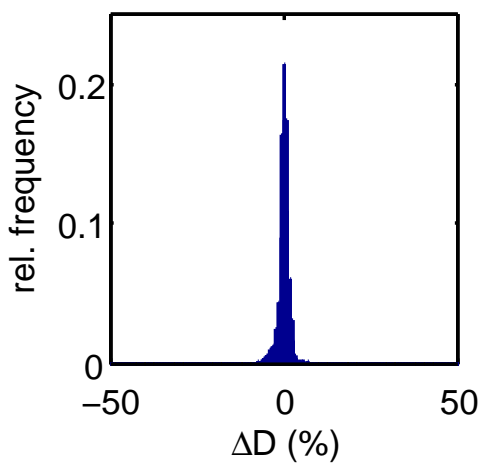

(b)

Figure 7. Relative difference histograms of untracked (a) and tracked (b) relative dose distribution.

Figure 7 integrates the relative dose maps of figure 6 into histograms; 7a shows the distribution of dose difference between static object (reference) and untracked object. The multimodal distribution shows two lateral side maxima caused by the comparatively long excursion plateaus of the motion trace. In figure $7 \mathrm{~b}$, the distribution morphs into a compact, mean-free Gaussian-like distribution with $\widehat{\sigma}(\Delta D)=1.38 \%$ using tracking. 


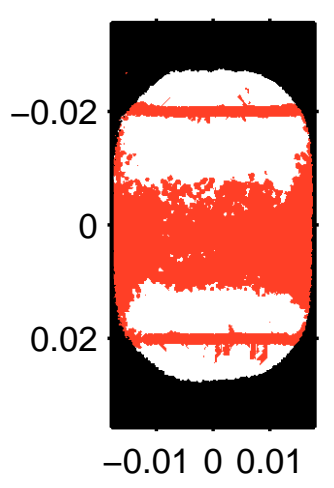

(a) untracked aperture

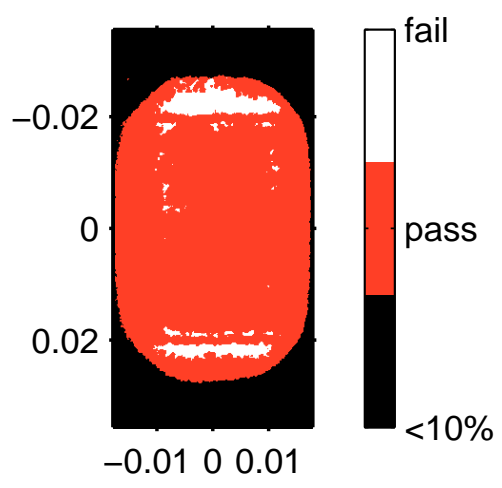

(b) tracked object

Figure 8. Thresholded $\gamma(1 \% 1 \mathrm{~mm})$ maps of films without (a) and with MLC tracking (b). The red area designates the region of $D_{r e l}>10 \%$ where the $\gamma$-test has passed, whereas white indicates points of failed test. Axis units in $m$.

In the $\gamma$-map of figure 8 , The $\gamma$-pass rate, extracted from the thresholded $(\gamma<1)$ maps of figure 8 changed from $49 \%$ for the untracked case, to $92 \%$ for the tracked case. The concentration of $\gamma$-failed points is in regions of large spatial dose gradients, i.e. around the location of the leaf tips. The pass area in both $\gamma$ evaluations at $y=0.02 m$ shows intersecting dose profiles, the reference dose and the dose accumulating during tracking being at the same level. Due to the ideally strong gradients with the reference aperture a pass region will be observed with a width solely dependent on the geometric component of the $\gamma$-statistics.

\section{Discussion}

For the dynamic experiments, the Agility MLC was driven in a continuous manner, avoiding retarding inertial effects. This resulted in a low MLC delay of $15.9 \mathrm{~ms}$ shown by indirect measurement. Although exhibiting significant variance, the phase featured linear phase behaviour in the sampled parameter field. Considering the real-time frame of $40 \mathrm{~ms}$, this small delay is likely to be caused by feed-forward terms in the MLC control system, imposing a ballistic extrapolation between adjascent control points of two consecutive cycles. These findings complement step-responses for the Varian Millenium MLC by (Poulsen et al. 2010) in which time delay was determined to be constant for a limited MLC adjustment step.

Aiming for potential MLC tracking in an MR-Linac, $2 D$ imaging rates are expected to be in a range of 2-4 frames per second; accordingly an upsampled, extrapolated object position variable in order to update the MLC in every control system cycle could yield a dosimetric advantage of low MLC latency compared step-wise MLC response. Furthermore, using $1 D$ navigator echoes, Stam et al. (2012) could get anatomy information every $15 \mathrm{~ms}$, which could be used as a fast surrogate tracking variable in combination with an organ correlation model. 
Compared to the low apparent MLC latency, current clinical position extraction modalities such as approximately 90ms for Calypso (Smith et al. 2009), 100ms for MRI (Ries et al. 2010) and > 160ms for $\mathrm{kV} / \mathrm{MV}$ imagers (Poulsen et al. 2010) contribute to the overall latency to a much higher extent. Notably, for the non-clinical tracking implementation with a customary camera, the most significant contributor to the overall delay of about $110 \mathrm{~ms}$ was the imaging API itself. The API delivered images with an average of $78.7 \mathrm{~ms}$, which can be explained by the buffer strategy of the frame capturing cascade of the OpenCV API (Schellen et al. 2013). It is however expected that this lag component can be be overcome in a clinical modality using real-time communications as demonstrated by Ries et al. (2010) and Crijns, Raaymakers \& Lagendijk (2012).

Knowing the MLC-inherent logging delay $\Delta T_{4}$ in addition to the MLC delay, dose can be accumulated retrospectively by knowing both temporal and geometric interplay of MLC and anatomy. The variance of $\Delta T_{4}$, although being extracted from only a single indirect measurement, is expected to be small, since the position subscription runs with a time frame of the Agility MLC control system.

Dosimetric deviations are distributed in a non-standard distribution for both tracking and static MLC applications. Qualitatively, both the relative differences and the $\gamma$-score show the performance improvement of tracking $(\gamma=92 \%)$ versus static $(\gamma=49 \%)$ MLC irradiation. Since the previously published, more relaxed $\gamma$ statistics with radii of as shown by Smith et al. (2009) $3 \mathrm{~mm} / 3 \%$ are intended to rate the outcome of the entire treatment chain, the stringent $\gamma(1 \mathrm{~mm} / 1 \%)$-statistics may be more suitable as a representative integrated quantifier for rating the tracking performance of the tracking-system in an isolated manner. Observed dosimetric deviations laterally, perpendicular to the leaf travel direction, is due to to radial anisotropy of the beam itself and the dosimetry setup, i.e. differing path lengths through the dosimetry set-up. The latter is adding to the fact that retrospective dose accumulation cannot solely be computed with a simple convolution kernel constructed by system delay, but has to account for the beam characteristics, e.g. by incorporating the aperture and anatomy position into the treatment planning system (TPS) for retrospective dose calculation published by Poulsen et al. (2012) and clinically used by Keall et al. (2014). This is especially true in flattening filter-free applications. However, while knowledge about machine geometry is comparatively easy to obtain knowing the timing parameterization, more details about tissue position over time has to be integrated into the process either by fast $3 D$ imaging techniques or precise motion models using surrogates. Recently, Bjerre et al. (2013) and Brix et al. (2014) could successfully employ MRI to rapidly infer $3 D$ anatomy motion.

For applying tracking in the second dimension perpendicular to the leaf-motion, on-line sequencing needs to be elaborated. Also, the dependency of the MLC dynamics on gantry and collimator angle needs further investigation. As shown within this paper, position prediction filters will be needed to be further investigated in order to keep the MLC in the presented continous motion and to compensate for the potentially variable system delay. 


\section{Conclusion}

The tested Agility MLC system showed notably low latency times when driven in a continuous manner; extracted timing characteristics indicates good MLC tracking performance with regards to commonly employed imaging and surrogate positioning techniques. Counteracting bottlenecks in tracking cascades will thus have to emphasize on fast and responsive imaging techniques. The shown phase linearity enables generic parameterization of predictive algorithms. An adaptive framework for dynamic multileaf collimator (dMLC) tracking employing the Agility MLC could succesfully be employed and first dosimetric experiments based on in-vivo liver motion showed expectably improved dosimetry performance both in relative dosimetry and in a $\gamma$-sense.

\section{Acknowledgments}

This work was supported by research funds from Elekta Ltd., UK. We appreciate their efforts in providing experimental research versions of machine firmware which enabled this work. 


\section{References}

Berbeco R I, Mostafavi H, Sharp G C \& Jiang S B 2005 Phys. Med. Biol. 50(19), 4481-90.

*http://www.ncbi.nlm.nih.gov/pubmed/16177484

Bjerre T, Crijns S, af Rosenschöld P M, Aznar M, Specht L, Larsen R \& Keall P 2013 Phys. Med. Biol. 58(14), 4943-50.

*http://www.ncbi.nlm.nih.gov/pubmed/23807514

Bolme D, Beveridge J R, Draper B A \& Lui Y M 20102010 IEEE Comput. Soc. Conf. Comput. Vis. Pattern Recognit. pp. 2544-2550.

*http://ieeexplore.ieee.org/lpdocs/epic03/wrapper.htm?arnumber $=5539960$

Bradski G 2000 Dr. Dobbs J. 25(11), 120-126.

Brix L, Ringgaard S, Sø rensen T S \& Poulsen P R 2014 Med. Phys. 41(4), 042302. *http://www.ncbi.nlm.nih.gov/pubmed/24694152

Cerviño L I, Chao A K Y, Sandhu A \& Jiang S B 2009 Phys. Med. Biol. 54(11), 3529-41.

*http://www.ncbi.nlm.nih.gov/pubmed/19443952

Crijns S P M, Kotte A N T J, Lagendijk J J W \& Raaymakers B W 2012 in 'Proc. Int. Soc. Magn. Reson. Med.' p. 987.

Crijns S P M, Raaymakers B W \& Lagendijk J J W 2012 Phys. Med. Biol. 57(23), 7863-72.

*http://www.ncbi.nlm.nih.gov/pubmed/23151821

Davies G A, Clowes P, Bedford J L, Evans P M, Webb S \& Poludniowski G 2013 Phys. Med. Biol. 58(13), 4643-57.

*http://www.ncbi.nlm.nih.gov/pubmed/23780400

Falk M, Larsson T, Keall P, Chul Cho B, Aznar M, Korreman S, Poulsen P \& Munck Af Rosenschold P 2012 Med. Phys. 39(3), 1588-94.

*http://www.pubmedcentral.nih.gov/articlerender.fcgi?artid=3306442\&tool=pmcentrez\&rendertype=abstract

Giraud P, Yorke E, Jiang S, Simon L, Rosenzweig K \& Mageras G 2006 Cancer Radiother. 10(5), 269-

82.

*http://www.ncbi.nlm.nih.gov/pubmed/16875860

Horn B K \& Schunck B G 1981 Artif. Intell. 17(1-3), 185-203.

*http://linkinghub.elsevier.com/retrieve/pii/0004370281900242

Keall P J, Colvill E, O'Brien R, Ng J A, Poulsen P R, Eade T, Kneebone A \& Booth J T 2014 Med.

Phys. 41(2), 020702.

*http://www.pubmedcentral.nih.gov/articlerender.fcgi?artid=3977852\&tool=pmcentrez\&rendertype=abstract

Koolwal K 2009 RTLWS11.

*https://lwn.net/images/conf/rtlws11/papers/proc/p19.pdf

Lagendijk J J W, Raaymakers B W \& van Vulpen M 2014 Semin. Radiat. Oncol. 24(3), 207-209.

*http://www.ncbi.nlm.nih.gov/pubmed/24931095

Low D A, Harms W B, Mutic S \& Purdy J a 1998 Med. Phys. 25(5), 656-61.

*http://www.ncbi.nlm.nih.gov/pubmed/9608475

Poulsen P, Cho B, Sawant A, Ruan D \& Keall P 2010 Med. Phys. pp. 4998-5005.

*http://scitation.aip.org/content/aapm/journal/medphys/37/9/10.1118/1.3480504

Poulsen P, Schmidt M \& Keall P 2012 Med. Phys. 39(October), 6237-6246.

*http://scitation.aip.org/content/aapm/journal/medphys/39/10/10.1118/1.4754297

Raaymakers B W, Lagendijk J J W, Overweg J, Kok J G M, Raaijmakers a J E, Kerkhof E M, van der

Put R W, Meijsing I, Crijns S P M, Benedosso F, van Vulpen M, de Graaff C H W, Allen J \&

Brown K J 2009 Phys. Med. Biol. 54(12), N229-37.

*http://www.ncbi.nlm.nih.gov/pubmed/19451689

Ries M, de Senneville B D, Roujol S, Berber Y, Quesson B \& Moonen C 2010 Magn. Reson. Med.

64(6), 1704-12.

*http://www.ncbi.nlm.nih.gov/pubmed/20878763

Ruan D 2010 Phys. Med. Biol. 55(5), 1311-26. 
*http://www.ncbi.nlm.nih.gov/pubmed/20134084

Ruan D, Kupelian P \& Low D a 2011 Cancer J. 17(3), 155-8.

*http://www.ncbi.nlm.nih.gov/pubmed/21610468

Sawant A, Smith R L, Venkat R B, Santanam L, Cho B, Poulsen P, Cattell H, Newell L J, Parikh P \& Keall P J 2009 Int. J. Radiat. Oncol. Biol. Phys. 74(2), 575-82.

*http://www.ncbi.nlm.nih.gov/pubmed/19327907

Schellen J, Dernehl C \& Kowalewski S 2013 in 'IMAV' pp. 17-20.

Smith R L, Sawant A, Santanam L, Venkat R B, Newell L J, Cho B C, Poulsen P, Catell H, Keall P J \& Parikh P J 2009 Int. J. Radiat. Oncol. Biol. Phys. 74(3), 868-75.

*http://www.ncbi.nlm.nih.gov/pubmed/19394159

Stam M K, Crijns S P M, Zonnenberg B A, Barendrecht M M, van Vulpen M, Lagendijk J J W \& Raaymakers B W 2012 Phys. Med. Biol. 57(21), 6797-805.

*http://www.ncbi.nlm.nih.gov/pubmed/23032581

Tacke M B, Nill S, Krauss A \& Oelfke U 2010 Med. Phys. 37(2), 753.

*http://scitation.aip.org/content/aapm/journal/medphys/37/2/10.1118/1.3284543

Yun J, Wachowicz K, Mackenzie M, Rathee S, Robinson D \& Fallone B G 2013 Med. Phys. 40(5), 051718.

*http://www.ncbi.nlm.nih.gov/pubmed/23635266 